\title{
A New Approach for Beaconless Geographical Routing in Wireless Sensor Networks
}

\author{
OMID YAZDANI ${ }^{1}$ and SHAPOOR GOLBAHAR HAGHIGHI ${ }^{2}$ \\ ${ }^{1,2}$ Affiliation, Department Of Computer And Electrical Engineering, Shiraz University, Shiraz, Iran \\ E-mail: 'omid.yazdani563@gmail.com, 29olbahar@shirazu.ac.ir
}

\begin{abstract}
Lately geographic routing algorithms applicable for large scaled in sensor network. In existing geographic routing algorithms, the packet is sent to neighbor that closest to destination by using a greedy forwarding scheme. Whereas to access the neighbor's position requires that a mechanism beaconing periodically. So in the sensor network with highly dynamic topology the communication overhead is increased. Therefore, many beaconless geographic routing has been introduced recently. In this paper a novel approach for beaconless geographic routing (NBGR) is proposed. NBGR acts in two modes: a main mode and recovery mode. In the main mode three kinds of control message RTS, CTS and SELECT is used. Thus a forwarder node broadcast an RTS and sets a timer. The neighbors in the positive progress area (PPA) that receive the RTS set a timer with appropriate delay function independently. So that the closest neighbor to destination responds first. This process is continued until the packet has received to node that don't have any neighbor in its PPA, then routing shifted into recovery mode. In Recovery mode the packet is sent to the destination by traversing on the face of planar graph such as a relative neighborhood graph (RNG) or Gabriel graph (GG). We show in NBGR the packet delivery ratio is improved than same algorithms such as BLR and $\mathrm{CBF}$ as well, according to the new delay function the energy of nodes are applied in a balanced way and the possibility of collision of CTS send back the forwarder node is decreased considerably. Also in NBGR the based on the selections from the suitable forwarding area the probability of the void area problem is minimized.
\end{abstract}

Keywords: Wireless Sensor Networks, Geographical Routing, Forwarding Area, Delay Function, Recovery Mode.

\section{INTRODUCTION}

Wireless sensor networks (WSNs) consist of many sensor nodes with low Consumption power and limited memory. Scalability and energy saving are the most important challenges of routing algorithms in WSNs.

Conventional routing algorithms because of the need to route establishment and maintaining path to each node, are fully proactive. Therefore, in WSNs due to limited of resources such as battery power, communication bandwidth, processing capability and memory of the nodes, it isn't useful for large networks [1].

Recently, Geographic routing is introduced as a popular localized and scalable routing for wireless sensor networks (WSN) [1]. The most commonly scheme used in the geographic routing is to be called greedy routing. At every step in greedy forwarding each node sends a packet to the neighbor who is nearest to the destination based only its own position, neighbor and destination position. The position of the source node and final destination are achieved by Global Positioning System (GPS) or network localization algorithms. Also location information of neighbors is periodically exchanged by beacons. Therefore, geographic routing is generally considered to be scalable and applicable to large networks.

Although in traditional geographical routing the communication overhead is reduced considerably, but in the sensor networks with dynamic topology, location information of nodes is out-of-date very fast, so each node to obtain its exact position needed for the exchange of beacons to neighbors and it results in consume network resources. 
To overcome the disadvantages of geographic routing algorithms in the highly mobile networks, beaconless geographical routing protocols have been proposed.

In Beaconless geographical routing algorithms, each node according to its own position and destination forwards the packets to destination through the use of a mechanism timer-based without requiring to position information of neighbors.

So to overcome the disadvantages of geographic routing algorithms with highly dynamic network, beacon less geographic routing protocols have been proposed [2].

In summary, advantages of beaconless geographical routings are as follows:

1-forwarding the packets without using position information of neighbors will cause a decrease of communication overhead.

2-in sensor networks with dynamic topology which the mobility of nodes is high, position of neighbors is less accurate which this problem is solved in beacon less geographical routing algorithms.

In this paper, we propose a new method for beaconless geographical routing algorithms (NBGR). In NBGR algorithm compared with another scheme chose a typical forwarding area, to find the neighbors as a next hop, replace of sector 60 or Reuleaux triangle. Because the probability of the void in this forwarding area is smallest between the three areas also having a neighbor closer to the destination in said region is high (it is proved that in []) which is leading to a high packet delivery ratio than other algorithms like BLR and CBF finally. Also in proposed algorithm a new delay function is introduced so the number of simultaneously sending in the same time is low and implies to reduce collision or packet duplication. Another benefit of NBGR is when the nearest neighbor to the destination than the forwarder node does not exist (problem local minimum) in this case, Algorithm is switched to recovery mode unlike CBF algorithms.

The rest of this paper is comprised of the following parts: in the next part assumptions are used in the rest of the paper will describe. In section 3 and 4 we define a different forwarding area and appropriate new delay function. In section 5 we explain the greedy forwarding approach in the main mode. To solve the problem of void area the recovery mode is presented in section 6 . In section 7 we state how decreases the packet duplication in proposed algorithm and Section 8 provides numerical and simulation results finally.

\section{ASSUMPTIONS}

1-position of nodes is achieved by GPS or localization algorithms.

2-the source node knows the position of the final destination and put it in the header of forwarding packets.

3-transmission range of all nodes is similar.

4-Tmax parameter is specified for all nodes in the network.

5 -in recovery mode network unit graph and adjacent neighborhood graph is being used.

6- MAC layer will assume ideal without any packet loss because of the collision.

\section{FORWARDING AREA}

Forwarding area is described as the area where potential forwarding nodes as a next hop exist in. Generally three kinds of forwarding area is introduced that has been shown in figure (1) [3].

\subsection{Positive Progress Area (PPA)}

PPA is the intersection of two circles: one the circle indicate the transmission range of the forwarder node and another circle is centered to destination with radius equal to distance between the sender node and the destination [3]. It is illustrated in figure (1) (OSPW). The distance nodes within in PPA to destination shorter than forwarder node and becomes a potential candidate node for receiving the packet from the source node.

\subsection{Maximum Communication Area (MCA)}

The largest area that is each node in transmission range of each other. So this area is a circle with diameter SW as shown in figure (1). The nodes in MCA can hear each other. The size of MCA invariable against the PPA.

\subsection{0-Degree Radian Area (DRA):}

DRA included 30 degree radial area around line SD that is a connected line a forwarder node to a destination so that its angle bisector. DRA illustrated by SEWM in figure (1).

Thus, in MCA \& DRA area every node be able to hear each other.

We apply the PPA area by the following reasons;

1. PPA area larger than two another area

2. The probability of void in the PPA is smallest among the three areas [3].

The second property is prove that [3] also it is an upper bound for probability of vid in PPA has been derived [4]. 
As it has been mentioned [3] in this forwarding area the probability of void region is lower than sector 60 or circle with diameter (r). Because it is obvious that the probability of having a neighbor closer to the destination in all the PPA, is more than sector 60 or circle with diameter (r) in CBF and BLR algorithms.

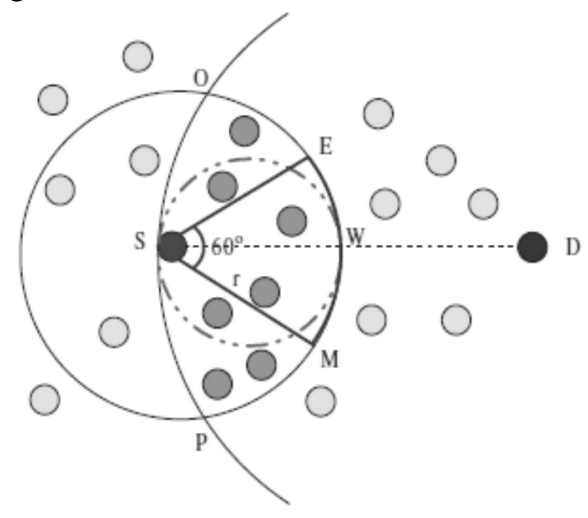

Fig. 1.Diffrent Forwarding Area [3]

\section{DELAY FUNCTION}

In NBGR we use the delay function:

$\operatorname{Tmax}[\mathrm{w} 1(1-\mathrm{p}(\mathrm{d}-\mathrm{c}) /(\mathrm{r}) 2)+\mathrm{w} 2 \mathrm{v}]$

$\mathrm{r}$ : shows the transmission range of nodes.

Tmax: shows maximum delay of forwarder nodes.

d: shows distance between sender node to destination.

P: shows projection of neighbor on the connected line of sender node to destination.

c: shows distance between neighbor nodes to destination.

$\mathrm{v}$ : shows a random number interval $(0,1)$.

w1, w2: shows weighted coefficients so $\mathrm{w} 1+\mathrm{w} 2=1$

The term $w 2 v$ in the delay function for avoiding the packets is to be sent simultaneously by nodes. This property avoids the packet duplication in the network.

Therefore, according to the delay function, a node from among its neighbors in the forwarding area will be chosen that has more progress and nearer distance to destination.

Generally in NBGR a packet is to be sent to the destination in two mod; main mode and recovery mode. The Forwarder node sends a packet to the closest neighbor to the destination by greedy routing in main mode when the greedy forwarding fails the routing switch to recovery mode. In the next section the main mode will describe in detail.

\section{MAIN MODE}

In the main mode, at first the source node having a packet, locates the destination positions with its own position in the RTS message. Then, it broadcasts to neighbors and, simultaneously, sets its timer to Tmax. Tmax should be taken so as long as the time needed for getting a packet from its farthest neighbor.

On the other side, Neighbors in the positive progress area (PPA) when receiving the RTS, set a timer according to the mentioned delay function in the previous section. Also Neighbors in negative progress area (NPA) discard it.

Due to the delay function, the node closer to the destination, It must have expired its timer sooner than all the other neighbors in PPA and, then, it broadcasts the CTS included its position (The contention stage). Whenever the other neighbors overhear the CTS message find out there is another node which is closer to the destination and then they will cancel their timer (The suppression stage). When the CTS is received to the source node, it will conclude that there is a candidate node as a next hop in PPA and now the source node can cancel its timer. After that, the source node sends out a SELECT message which includes the position of a candidate node that sends CTS before all. Some of forwarder node's neighbors which did not receive CTS, yet, after receiving the SELECT message now check their position and then cancel its timer. So they avoid from sending another CTS. Eventually the source node unicast the data packet to the node that receive CTS earlier. Also, if the source node receives more than one CTS from neighbors, then it would select a node that is closer to the destination (according to their position). This algorithm will be continued like this until the packet receives to the destination finally.

\section{RECOVERY MODE}

If the source node does not receive any CTS from its neighbors than it would conclude there is no neighbor in its PPA. So, to resolve this problem the routing will be switched to recovery mode. In recovery mode, at first the source node broadcast a small packet to its neighbors in NPA and the neighbors also put their position in another packet and send it. Now, the source node constructs a local planar sub graph by using location information of the neighbors that is called relative neighborhood graph (RNG). Therefore, Packet is to be sent to 
neighbors by using the Right Hand rule. This rule states that when arriving at node $\mathrm{x}$ from node $\mathrm{y}$, the next edge traversed is the next on sequentially counterclockwise about $\mathrm{x}$ from the edge (x, y) [6]. The header of sent packet in recovery mode, like GPRS, includes destination position, the position of a node which in it routing has changed to recovery mode and also routing mode. Routing is continued until the packet is received to the node that closer to the destination than the first node which routing has been switched to recovery mode.

\section{PACKET DUPLICATION}

Generally, in the geographical routing algorithms, the packet duplication may have happened, according to different reason such as follows:

1. Although, in traditional beaconless geographical routing the probability forwarding CTS's in the same time with other neighbors is high because of they have equal delay time, but considerably in proposed algorithm this possibility would be decreased.

2. Another reason for packet duplication due to doesn't enough time for suppression of nodes. So more than one CTS receive to source node. Therefore the source node unicast a data to node that closer to the destination

3. Finally, if Source node's neighbors haven't been locate in the transmission range of nodes that forwarding a CTS earlier, the source node will broadcast a select message to neighbors and then the neighbors don't receive a CTS formerly, cancel its timer now.

In the next chapter, the packet delivery ratio NBGR, CBF and BLR based on the number of nodes and nodes transmission range will be compared each other.

\section{SIMULATION}

Since the packet delivery ratio is an important parameter to determine the efficiency of routing algorithms. In this section as can be seen packet delivery ratio of NBGR algorithm is improved compared to other algorithms such as BLR and CBF. Because whenever a forwarder node doesn't have any neighbor in its PPA switched to recovery mode. In figures (2) \& (3) packet delivery ratio in terms of number of nodes and the various transmission range is demonstrated.

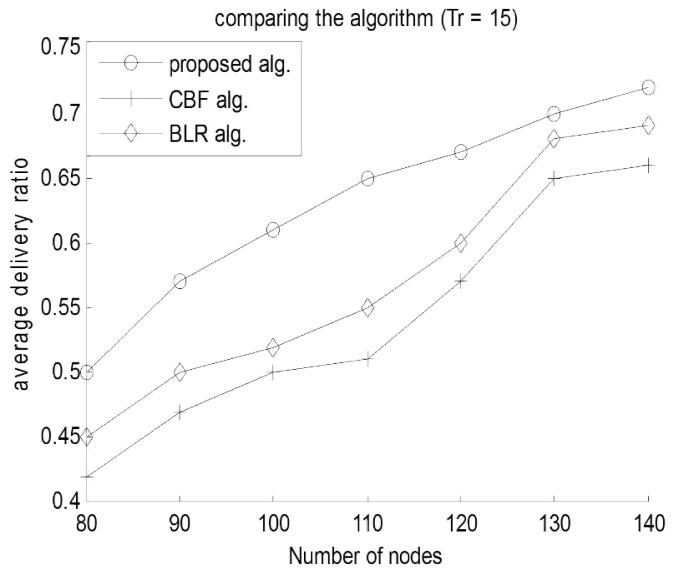

Fig.2. Comparing The NBGR With BLR \& CBF Algorithms

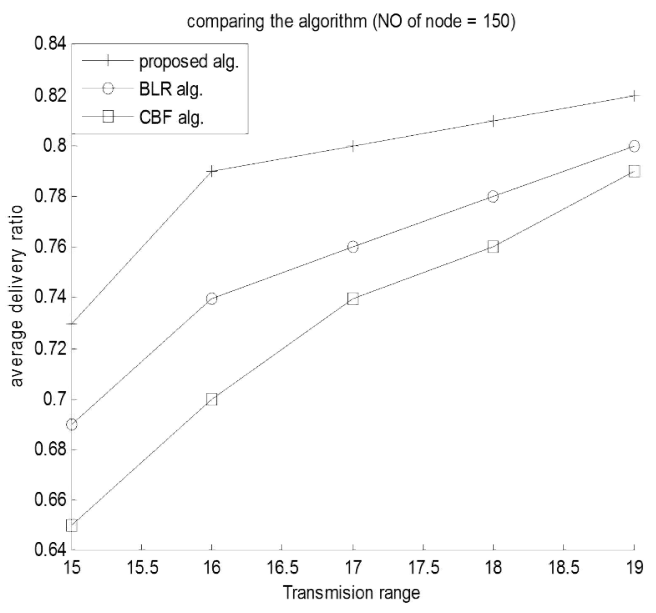

Fig.3. Comparing The NBGR With BLR \& CBF Algorithms

\section{CONCLUSION}

In this paper a new approach for Beaconless geographic routing algorithms is proposed. The traditional beaconless greedy scheme has two problems. Firstly the neighbors in PPA that have set a contention timer simultaneously responses. Secondly the nodes not in their transmission range of each other in the forwarding area could not suppress each other. Both cases cause packet duplication. In proposing algorithm the first problems are solved by adding the random value to delay function. A solution of second problem's is after that a sender node received a CTS broadcast the SELECT message to neighbors which did not receive CTS yet. Therefor by these considerations 
the load traffic is balanced. Moreover, as we have seen in simulation because of the void handling mechanism the packet delivery ratio is improved.

\section{REFERENCES}

[1] A. M. Popescu, I.G. Tudorache, B. Peng, and A.H. Kemp. "Surveying Position Based Routing Protocols for Wireless Sensor and Adhoc Networks", International Journal of Communication Networks and Information Security (IJCNIS), Vol. 4, No. 1, Apr 2012

[2] A.Kaya, A.Zengin and H.Ekiz, Comparision Of The Beacon-Less Geographic Routing Protocols In Wireless Sensor Networks, IEEE, Turkey, 2011

[3] D.Chen, J.Deney, K.Varashney, A State-Free Data Delivery Protocol For Multihop Wireless Sensor Networks, IEEE, 2005

[4] H. Füßler, J. Widmer, M. Mauve, and H. Hartenstein. "A Novel Forwarding Paradigm for Position-Based Routing (with Implicit Addressing) ", IEEE Computer Comm, pp. 194-200., 2003

[5] M. Heissenbüttel, T. Braun, Th. Bernoulli and M. Wächli. " BLR: Beacon-Less Routing Algorithm for Mobile Ad-Hoc Networks", Elsevier's Computer Communications Journal (ECC), vol. 27, 2004, no.11

[6] B. Karp and H.T. Kung. "GPSR: Greedy Perimeter Stateless Routing for wireless networks", Proc. MOBICOM pp.243-254, Aug. 2000. 